\title{
Efecto de la relación sustrato-inóculo sobre el potencial bioquímico de metano de biorresiduos de origen municipal
}

\author{
Effect of Substrate-Inoculum Ratio on the Biochemical Methane Potential \\ of Municipal Biowastes
}

\author{
Parra-Orobio Brayan Alexis \\ Escuela de Ingeniería de Recursos Naturales y del Ambiente \\ Universidad del Valle \\ Correo:brayan.parra@correounivalle.edu.co \\ Torres-Lozada Patricia \\ Escuela de Ingeniería de Recursos Naturales y del Ambiente \\ Universidad del Valle \\ Correo:patricia.torres@correounivalle.edu.co \\ Marmolejo-Rebellón Luis Fernando \\ Escuela de Ingeniería de Recursos Naturales y del Ambiente \\ Universidad del Valle \\ Correo:luis.marmolejo@correounivalle.edu.co \\ Cárdenas-Cleves Lina Marcela \\ Escuela de Ingeniería de Recursos Naturales y del Ambiente \\ Universidad del Valle \\ Correo:lina.cardenas.cleves@correounivalle.edu.co
}

\author{
Vásquez-Franco Carlos \\ Escuela de Ingeniería de Recursos Naturales y del Ambiente \\ Universidad del Valle \\ Correo:carlos.vasquez@correounivalle.edu.co
}

\author{
Torres-López Wilmar Alexander \\ Escuela de Estadística \\ Universidad del Valle \\ Correo:alexandertor@gmail.com \\ Ordoñez-Andrade José Abdón \\ Departamento de Gestión del Agua Urbana \\ Universität Kassel \\ Correo: joseabdonordonez@hotmail.com
}

Información del artículo: recibido: abril de 2014, aceptado: enero de 2015

\section{Resumen}

En los países en desarrollo, los biorresiduos son la fracción predominante de los residuos sólidos municipales (RSM); los biorresiduos tienen un alto contenido de materia orgánica, característica que favorece su transformación a través de procesos biológicos como la digestión anaerobia (DA). En este estudio, mediante ensayos de potencial bioquímico de metano (PBM), se evaluó la influencia de la relación sustrato-inóculo (S/I) sobre la DA de biorresiduos de origen municipal (BOM), utilizando relaciones de 0.25 a $9 \mathrm{gSV}_{\text {sustrato }}{ }^{*} \mathrm{gSV}_{\text {inóculo }}{ }^{-1}$. Como inóculo se empleó lodo de un biodigestor anaerobio de una planta de tratamiento de aguas residuales domésticas. Se encontró que la relación S/I tiene efecto sobre la DA de los BOM $(\mathrm{p}<0.1)$, en donde la relación 0.25 produjo los mejores re-

\section{Descriptores:}

- biorresiduos

- digestión anaerobia

- potencial bioquímico de metano

- relación sustrato-inóculo 
sultados (producción de $176.19 \mathrm{mLCH}_{4}{ }^{*} \mathrm{gSV}^{-1} \mathrm{y}$ un índice de biodegradabilidad de $73.12 \%$, mientras que la relación de 9 generó los más bajos resultados (17.56 $\mathrm{mLCH}_{4}{ }^{*} \mathrm{gSV}^{-1}$ y $7.29 \%$, respectivamente). También se encontró que las relaciones $\mathrm{S} / \mathrm{I}$ menores de $2 \mathrm{gSV}_{\text {sustrato }}{ }^{*} \mathrm{gSV}_{\text {inóculo }}{ }^{-1}$ permiten un proceso adecuado; con valores mayores, el proceso se desestabiliza debido a la ocurrencia de procesos de acidificación por la acumulación de los AGV's que no logran estabilizarse por la baja capacidad buffer del sistema.

\begin{abstract}
Biowastes are the predominant fraction of municipal solid waste (MSW) of developing countries. Biowastes are characterized by a high content of organic matter; characteristics which facilitates its transformation through biological processes such as anaerobic digestion ( $A D)$. Using biochemical methane potential (PBM) assays, in this study we evaluated the influence of substrate-inoculum (S/I) ratio on the AD of biowaste from MSW. The S/I ranged between 0.25 to $9 \mathrm{gSV} V_{\text {substrate }}$ * $g S V_{\text {inoculum }}{ }^{-1}$. Sludge from an anaerobic digester of the domestic wastewater treatment plant located in Cali-Colombia, was used as inoculum. It were found that the $S / I$ ratio has an effect on the $A D(p<0.1)$. Best results was obtained with the ratio $0.25 \mathrm{gSV}_{\text {substrate }}{ }^{*} g S V_{\text {inoculum }}{ }^{-1}\left(176.19 \mathrm{mLCH}_{4}{ }^{*} \mathrm{~g} S V^{-1}\right.$ and a rate of $73.12 \%$ biodegradability), while $9 \mathrm{gSV} V_{\text {substrate }}{ }^{*} g S V_{\text {inoculum }}{ }^{-1}$ produced the lowest results (17.56 and $7.29 \%$ respectively). Furthermore, it was found that S/I ratios lower than $2 g S V_{\text {substrate }}{ }^{*} g S V_{\text {inoculum }}{ }^{-1}$ provided a suitable process. In the other hand, with $S / I$ ratios greater than 2, the process becomes unstable due to acidification processes, caused by accumulation of VFA's; those VFA's cannot be he stabilized due to the low buffering capacity of the system.
\end{abstract}

\section{Introducción}

La producción mundial de residuos sólidos municipales (RSM) es del orden de $2 \times 10^{9}$ ton $^{*}$ año $^{-1}$ y posiblemente para el año 2025 alcance $3 \times 10^{9}$ ton $^{*} a^{-1} o^{-1}$ (Charles et al., 2009), ya que una gran parte de ellos son biorresiduos de origen municipal (BOM), los cuales están constituidos por residuos de jardín, comida pre y postconsumo de las viviendas y de establecimientos comerciales como restaurantes y expendios de alimentos, excluyéndose residuos de agricultura, actividades forestales, estiércol animal, biosólidos u otros como textiles, papel y madera procesada (Oviedo et al., 2012). En el continente europeo y en el centro de Asia, la proporción de BOM alcanza $47 \%$ y en países en desarrollo varía entre 54 y $62 \%$ (TWB, 2012); en el caso colombiano, la proporción llega a 65\% (Oviedo et al., 2012).

Durante el transporte, manipulación y disposición de los BOM, se generan problemas como la presencia de polvo en el aire, emisión de gases tóxicos o de efecto invernadero-GEI, así como la producción de lixiviados que contribuyen negativamente al ambiente y a la salud (Filigrana et al., 2011). Sin embargo, la fracción predominantemente orgánica de los mismos, favorece su transformación mediante procesos biológicos tanto ae- robios como anaerobios (Angelidaki et al., 2009). Estos últimos cuentan con una tecnología que cada día gana más importancia en el contexto mundial, ya que permite cerrar ciclos de contaminación y al mismo tiempo generar un gran potencial de producción de energías limpias a partir de residuos líquidos (aguas residuales domésticas, industriales) y sólidos (BOM, lodos primarios y secundarios de PTAR) (Verma, 2002).

La digestión anaerobia (DA) de BOM está influenciada por diferentes factores, en donde la relación sustratoinóculo (S/I) es un factor clave para la optimización del proceso. A pesar de que en la literatura se mencionan diferentes valores recomendables, estos oscilan dependiendo de las características del sustrato y del inóculo, además del tipo de unidades usadas para su determinación, las cuales se pueden expresar en $\mathrm{gDQO}_{\text {sustrato }}$ * $\mathrm{gSV}_{\text {inóculo }}{ }^{-1} \mathrm{y} \mathrm{gSV}_{\text {sustrato }}{ }^{*} \mathrm{gSV}_{\text {inóculo }}{ }^{-1}$, esta última considerada la más utilizada (Angelidaki y Sanders, 2004); por esta razón, autores como Owen et al. (1978), Aquino (2007) y Lesteur et al. (2010) recomiendan definir para cada sustrato e inóculo la proporción que garantice la mayor producción de metano. Los rangos de valores recomendables oscilan entre menos de 0.5 (Verein Deutscher Ingenieure-VDI (2006) a 1 (Raposo et al., 2006). Por otro lado, las relaciones superiores a 2 pue- 
den presentar problemas de inhibición, ya sea por altas concentraciones de sólidos totales o ácidos grasos volátiles (AGV's) (Raposo et al., 2011).

En este estudio se evaluó a escala de laboratorio el efecto de la relación S/I sobre la DA de los BOM, mediante ensayos de Potencial Bioquímico de Metano (PBM).

\section{Materiales y métodos}

\section{Ubicación experimental}

Los ensayos se desarrollaron en el Laboratorio de Biotecnología Ambiental de la Universidad del Valle, Sede Meléndez (Cali), que se encuentra a una altitud de 970 $\mathrm{m}$ con una temperatura promedio $23.6^{\circ} \mathrm{C}$.

\section{Fase experimental}

\section{Caracterización del sustrato (BOM) y el inóculo}

Los BOM procedieron de la planta de manejo de residuos sólidos (PMRS) del municipio de Versalles, Valle del Cauca, en donde se realiza una gestión integral de los residuos sólidos municipales desde el año 1997, mediante actividades como separación en la fuente y recolección selectiva (Marmolejo, 2011). El programa de muestreo y caracterización de los BOM correspondió a 5 muestras y se realizó bajo las recomendaciones de Sakurai (2000).

La composición física gruesa y fina de los BOM se evaluó por categorías, con base en lo sugerido por Marmolejo (2011); la composición gruesa incluyó residuos de alimentos (procesados y sin procesar), papel y cartón, poda y jardín e impropios (plástico, metales, caucho, etcétera); la composición fina se asoció a los alimentos sin procesar como carbohidratos- $\mathrm{C}$, frutas no cítricas-FNC, frutas cítricas y semi-cítricas-FC, fibra y mezcla de minerales-FM, hierba- $\mathrm{H}$, semilla-S y otros; asimismo mezcla de materiales (alimentos de todas las categorías con alto grado de descomposición y de difícil identificación).

La caracterización fisicoquímica se realizó de acuerdo con ICONTEC (2004), APHA (2005) e ICONTEC (2009) en términos de las variables pH (Unidades), Alcalinidad total-AT y bicarbonática-ATB $\left(\mathrm{mgCaCO}_{3}{ }^{*} \mathrm{~L}^{-1}\right)$, Ácidos Grasos Volátiles $\left(\mathrm{mg}^{*} \mathrm{~L}^{-1}\right)$, Humedad (\%), Carbono oxidable y total (\%), $\mathrm{UV}_{254}\left(\mathrm{~cm}^{-1}\right)$, DQO total y filtrada $\left(\mathrm{mg}^{*} \mathrm{~L}^{-1}\right), \mathrm{DBO}_{5}\left(\mathrm{mg}^{*} \mathrm{~L}^{-1}\right)$, Nitrógeno total $(\%)$, Nitrógeno amoniacal $\left(\mathrm{mg}^{*} \mathrm{~L}^{-1}\right)$, Celulosa $(\%)$, Almidón $(\%)$, Lignina (\%), Extracto etéreo (\%), Proteínas (\%), Carbohidratos, (\%), Fibra cruda (\%), Hidrógeno (\%), Oxígeno (\%), Sólidos totales y volátiles $\left(\mathrm{mg}^{*} \mathrm{~L}^{-1}\right)$.
Para los ensayos de PBM, se utilizó como inóculo lodo del digestor anaerobio de la Planta de Tratamiento Aguas Residuales de la ciudad de Cali (PTAR Cañaveralejo-PTAR-C), el cual se caracterizó (5 muestras) en términos de las variables fisicoquímicas determinadas a los BOM, con excepción de hidrógeno y oxígeno. Las muestras de BOM e inóculo se conservaron a una temperatura no mayor a $4^{\circ} \mathrm{C}$ por periodos inferiores a siete días, previo al montaje de los ensayos según recomendación de Sandoval et al. (2007).

Los datos tanto de la composición física como de las características fisicoquímicas de los BOM y el inóculo, se procesaron empleando métodos estadísticos descriptivos (media, desviación estándar y coeficiente de variación). Finalmente, la fracción biodegradable (FB) de los BOM se determinó de acuerdo con lo sugerido por Espinosa et al. (2007), quienes relacionan el material vegetal recalcitrante o inerte presente en estos. Para ello, se utilizó la ecuación 1.

$F B=0.83-0.028 C L$

Donde FB es fracción biodegradable expresada sobre la base de la materia volátil (MV); 0.83 y 0.028 constantes empíricas y CL es el contenido de lignina de la MV expresada como un porcentaje en peso seco.

\section{Descripción del ensayo de PBM}

Previamente al ensayo de PBM, se retiró el material inerte (piedras, metal, carbón, hueso) y de lenta degradación (plástico, caucho y cuero) de las muestras de BOM, como lo recomiendan Mukherjee et al. (2008); posteriormente se sometieron a un proceso de trituración (Sharma et al., 1988), empleando una trituradora CB15 Waring Commercial a una velocidad de 15800 rpm durante un minuto (velocidad estándar del equipo), garantizando tamaños de partículas inferiores a $30 \mathrm{~mm}$.

La cuantificación de biogás se realizó por el método manométrico, empleando el Sistema OxiTop @ (figura 1 ), el cual es un equipo de monitoreo de presión que consta de un reactor de $250 \mathrm{~mL}$ con un cabezal de medición que se inserta en la "boca" de los reactores y un control que usa una interfase infrarroja para transferir los datos.

Los ensayos se realizaron a temperatura controlada $\left(30^{\circ} \mathrm{C} \pm 0.5^{\circ} \mathrm{C}\right)$ en una incubadora WTW TS 606-G/2-i, con agitación manual intermitente durante 40 días, realizando mediciones cada 6 horas (Aquino, 2007). El volumen útil empleado fue de $200 \mathrm{~mL}$, dejando un espacio libre de $50 \mathrm{~mL}$ para el almacenamiento del biogás que se 


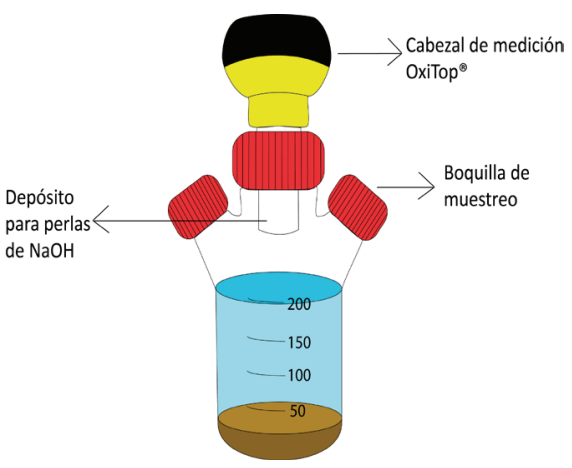

Figura 1. Unidad experimental para los ensayos PBM (Pabon et al., 2012)

produce, basados en las recomendaciones de Torres y Pérez (2010).

Para mantener condiciones estables en los ensayos PBM, se empleó una solución de macro y micronutrientes que parte del principio de que la técnica PBM es un método estandarizado que busca garantizar las condiciones más favorables que potencialicen la degradación anaerobia del sustrato (Owen et al., 1978); la solución utilizada fue la sugerida por Torres y Pérez (2010). Igualmente, se acondicionó el pH a 7.0 UND con una solución de $\mathrm{NaHCO}_{3}$ a 4\%. Para garantizar que la medición manométrica correspondiera predominantemente a metano, se capturó el dióxido de carbono a través de perlas de $\mathrm{NaOH}$ (Siefers, 2010), cuya composición de metano fue verificada mediante cromatografía de gases (Cromatógrafo GC2014).

Para determinar el volumen de metano a condiciones estándar (CE), se aplicaron las ecuaciones 2-12 (tabla 1 ) sugeridas por Aquino et al. (2007), Ortiz (2011) y Giménez et al. (2012), donde se considera la proporción de metano disuelto. De igual manera, se determinó el potencial bioquímicodemetanoteóricoyelíndicedebiodegradabilidad (\%B) del sustrato (Sobotka et al., 1983), con el fin de cuantificar la máxima producción y la facilidad de transformación de la materia orgánica mediante DA.

Para obtener diferentes valores de la relación S/I, en todas las unidades experimentales se mantuvo fija la concentración del inóculo, la cual correspondió a 1.5 $\mathrm{gSTV}^{*} \mathrm{~L}^{-1}$, valor recomendado por Field (1987) para ensayos PBM sin agitación continua. La concentración del sustrato se modificó en términos de gSTV en cada unidad experimental. La figura 2 muestra el esquema de las relaciones que se evaluaron. Cada unidad experimental contó con su respectivo duplicado y con un control (inóculo más agua destilada).

Para determinar el efecto de la relación S/I sobre el PBM, se aplicó un análisis de varianza (ANOVA) y una prueba de Tukey con $p<0.1$, en donde la variable respuesta es el PBM mediante el paquete estadístico R i386 3.0.2.

Finalmente, en cada una de las unidades experimentales se medió al inicio y al final del proceso la Alcalinidad (total y bicarbonática), AGV's y pH, lo cual se aprovechó durante los ensayos para tener un mayor número de elementos de análisis y comparación de los resultados de los estudios. Por lo anterior, se determinó el $\mathrm{I} \alpha$, que corresponde a la relación entre la alcalinidad bicarbonática y la alcalinidad total; mientras que el IB es la relación entre la alcalinidad debida a los AGV y la alcalinidad total y el Índice AI/AP es la asociación entre la alcalinidad debida a los AGV y la alcalinidad bicarbonática, todos se usaron como indicadores de la estabilidad del proceso (Torres y Pérez, 2008).

\section{Resultados y discusión}

\section{Caracterización de BOM e Inóculo}

La tabla 2 muestra la composición física de los BOM utilizados como sustrato en el proceso, en donde se ratifica que la fracción predominante son los residuos de comida $(93.13 \pm 1.75 \%)$. Estos resultados confirman las buenas prácticas de separación en la fuente por los usuarios y la eficiencia de los operarios de la PMRS en las actividades de separación y acondicionamiento de los BOM. Según Mukherjee et al. (2008), estas prácticas minimizan la presencia de compuestos de lenta biodegradación que pueden retardar la DA de los BOM y tener incidencia sobre la calidad de los productos del proceso (biogás y material estabilizado).

Los valores de la desviación estándar (DE) indican que los resultados están distribuidos adecuadamente con respecto al promedio de los datos totales para cada categoría; sin embargo, el coeficiente de variación (CV), presentó una alta oscilación, lo que concuerda con lo reportado por Grajales (2012), quien asoció este comportamiento a la presencia esporádica de alimentos procesados y residuos de poda y jardín en los BOM.

La tabla 3 presenta los datos de la subcategoría de alimentos sin procesar, relacionado con la composición fisica fina de los BOM. Se puede apreciar que predominan los carbohidratos y otros, seguidos por la categoría de frutas cítricas y semicítricas, resultados que coinciden con caracterizaciones previas (Oviedo et al., 2013) y con lo presentado por Parfitt et al. (2010) quienes caracterizaron la composición de los residuos de alimentos en naciones europeas y asiáticas, en donde encontraron que predominan los materiales como frutas frescas y vegetales (estos últimos contenidos en los carbohidratos y en la fibra y mezcla de minerales de los BOM caracterizados). 
DOI: https://doi.org/10.1016/j.riit.2015.09.004

Parra-Orobio B.A., Torres-Lozada P., Marmolejo-Rebellón L.F., Cárdenas-Cleves L.M., Vásquez-Franco C., Torres-López W.A., Ordoñez-Andrade J.A.

Tabla 1. Ecuaciones para determinar el PBM a través del método monométrico

\begin{tabular}{|c|c|c|c|}
\hline \multicolumn{2}{|l|}{ Ecuación } & \multirow[b]{2}{*}{$\mathrm{n}_{\mathrm{CH} 4}:$ Moles de $\mathrm{CH}_{4}(\mathrm{~mol})$} & \multirow[t]{2}{*}{ Finalidad } \\
\hline$n_{C H 4}=\frac{\Delta P^{*} V l}{R^{*} T_{e}}$ & (2) & & \\
\hline$V_{C H 4} C E=\frac{n_{C H 4}{ }^{*} R^{*} T_{C E}}{P_{C E}}$ & (3) & $\begin{array}{l}\Delta \mathrm{P}: \text { Incremento de la presión en el OxiTop } \\
(\mathrm{atm})\end{array}$ & $\begin{array}{l}\text { 2-3 Determinar el volúmen de metano a } \\
\text { condiciones estándar }\end{array}$ \\
\hline$H_{C H 4}=10^{\left(\frac{-675.74}{T e}+6.88\right)}$ & (4) & Vl: Volumen libre (L) & \\
\hline$X_{\mathrm{CHAdisuelto}}=\frac{\Delta P}{\mathrm{H}_{\mathrm{CH}_{4}}}$ & (5) & $\begin{array}{l}\text { R: Constante de los gases ideales } \\
\left(\operatorname{atm}^{*} \mathrm{~L}^{*} \mathrm{~K}^{-1 *} \mathrm{~mol}^{-1}\right)\end{array}$ & \\
\hline$M_{C H 4 d i s u e l t o}=\frac{M_{H 2 O} * X_{C H 4 d i s u e l t o}}{1-X_{C H 4 d i s u e l t o}}$ & (6) & $\mathrm{T}_{\mathrm{e}}:$ Temperatura del experimento $(\mathrm{K})$ & \\
\hline$n_{\text {CHAdisuelto }}=M_{\text {CHAdisuelto }}{ }^{*} V_{u}$ & (7) & $\begin{array}{l}\mathrm{V}_{\mathrm{CH} 4} \mathrm{CE} \text { : Volumen de } \mathrm{CH}_{4} \text { condiciones } \\
\text { estándar }(\mathrm{L})\end{array}$ & $\begin{array}{l}\text { 4-8. Determinar volumen de metano } \\
\text { disuelto }\end{array}$ \\
\hline$V_{\text {CHAdisuelto }} C E=\frac{n_{\text {CH4disuelto }}{ }^{*} R^{*} T e}{\Delta P}$ & (8) & $\mathrm{T}_{\mathrm{CE}}:$ Temperatura a condiciones estándar (K) & \\
\hline$V_{\mathrm{TCH} 4 \mathrm{CE}}=V_{\mathrm{CH} 4 \mathrm{CE}}+V_{\mathrm{CH} 4 d \text { disuelto }} \mathrm{CE}$ & (9) & $\mathrm{P}_{\mathrm{CE}}:$ Presión a condiciones estándar (atm) & \\
\hline$P B M=\frac{V_{T C H 4 C E}}{g S V}$ & $(10)$ & $\mathrm{H}_{\mathrm{CH} 4}$ : Constante de Henry para el $\mathrm{CH}_{4}(\mathrm{~atm})$ & $\begin{array}{l}\text { 9. Determinar volumen total de metano } \\
\text { en condiciones estándar }\end{array}$ \\
\hline$P B M_{\text {teorico }}=\frac{22.4\left(\frac{n}{2}+\frac{a}{8}-\frac{b}{4}-\frac{3 C}{8}\right)}{12 n+a+16 b+14 c}$ & $(11)$ & $\mathrm{X}_{\mathrm{CH} 4 \text { disuelto }}:$ Fracción molar de $\mathrm{CH}_{4}$ disuelto & $\begin{array}{l}\text { 10. Determinar el Potencial Bioquímico } \\
\text { de Metano }\end{array}$ \\
\hline \multirow{13}{*}{ 12. $\% \boldsymbol{B}=\frac{P B M}{P B M_{\text {teórico }}}$} & & $\begin{array}{l}\mathrm{M}_{\mathrm{CH} \text { disuelto }} \text { : Concentración molar del } \mathrm{CH}_{4} \\
\text { disuelto }\left(\mathrm{mol}^{*} \mathrm{~L}^{-1}\right)\end{array}$ & $\begin{array}{l}\text { 11. Determinar el Potencial Bioquímico } \\
\text { de Metano Teórico a CE }\end{array}$ \\
\hline & & $\mathrm{M}_{\mathrm{H} 2 \mathrm{O}}$ : Concentración molar del agua $\left(\mathrm{mol}^{*} \mathrm{~L}^{-1}\right)$ & \\
\hline & & $\mathrm{n}_{\mathrm{CH} 4 \text { disuelto }}:$ Moles de $\mathrm{CH}_{4}$ disuelto $(\mathrm{mol})$ & \\
\hline & & $\mathrm{V}_{\mathrm{u}}:$ Volumen útil de reactor $(\mathrm{L})$ & \\
\hline & & $\mathrm{V}_{\mathrm{CH} \text { disuelto }} \mathrm{CE}$ : Volumen de $\mathrm{CH}_{4}$ disuelto (L) & \\
\hline & & $\begin{array}{l}\mathrm{V}_{\mathrm{TCH} 4} \mathrm{CE} \text { : Volumen total de } \mathrm{CH}_{4} \text { condiciones } \\
\text { estándar }(\mathrm{L})\end{array}$ & \\
\hline & & $\begin{array}{l}\text { PBM: Potencial Bioquímico de Metano } \\
\left(\mathrm{L}^{*} \mathrm{gSV}^{-1}\right)\end{array}$ & $\begin{array}{l}\text { 12. Determinar el Índice de } \\
\text { Biodegradabilidad }\end{array}$ \\
\hline & & gSV: SV iniciales del sustrato (gSV) & $\begin{array}{l}\text { 2- 3. Determinar el volumen de metano a } \\
\text { condiciones estándar }\end{array}$ \\
\hline & & n: Número de moles de carbono & \\
\hline & & a: Número de moles de hidrógeno & \\
\hline & & b: Número de moles de oxígeno & \\
\hline & & c: Número de moles de nitrógeno & \\
\hline & & \%B: Índice de biodegradabilidad & \\
\hline
\end{tabular}

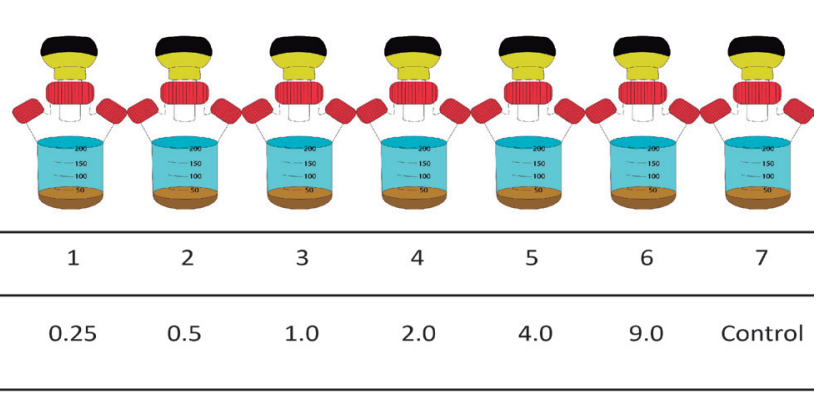

Figura 2. Representación esquemática de la unidad experimental 
Tabla 2. Composición física gruesa de los BOM

\begin{tabular}{cccc}
\hline \multicolumn{2}{c}{ Categoría (\%) } & Media & CV (\%) \\
\hline \multirow{2}{*}{ Residuos de comida } & Alimentos sin procesar & $91.25 \pm 3.25$ & 3.55 \\
\cline { 2 - 4 } & Alimentos procesados & $1.88 \pm 1.66$ & 88.50 \\
\hline Papel y Cartón & $1.27 \pm 0.37$ & 22.22 \\
Poda y Jardín & $1.42 \pm 0.47$ & 50.30 \\
Impropios & $3.64 \pm 1.68$ & 37.93 \\
\hline
\end{tabular}

Tabla 3. Categorías de los alimentos sin procesar (composición física fina de los BOM)

\begin{tabular}{lrr}
\hline \multicolumn{1}{c}{ Categoría $(\%)$} & \multicolumn{1}{c}{ Media } & \multicolumn{1}{c}{ CV } \\
\hline Carbohidratos & $34.45 \pm 10.97$ & 31.85 \\
Frutas no cítricas & $4.89 \pm 2.77$ & 56.51 \\
Frutas cítricas y semi cítricas & $13.73 \pm 7.34$ & 53.50 \\
Fibras y minerales & $7.85 \pm 3.13$ & 39.86 \\
Hierbas & $0.31 \pm 0.35$ & 113.86 \\
Semillas & $0.16 \pm 0.29$ & 183.96 \\
Otros & $33.89 \pm 16.13$ & 47.58 \\
\hline
\end{tabular}

La tabla 4 muestra la caracterización físico-química de los BOM e inóculo.

En general, los valores de $\mathrm{pH}, \mathrm{AT}, \mathrm{ATB}$ y AGV's son típicos de residuos rápidamente acidificables, como lo evidenciaron otros autores como Pesta (2007) y Li et al. (2010) en estudios de DA de BOM. Los bajos valores de pH están ligados a la descomposición de los BOM, que causa un aumento de la producción de $A G V^{\prime}$ s y que a su vez, es consistente con la ausencia de ATB, por lo tanto, surge la necesidad de acondicionar los BOM con un alcalinizante que aporte la capacidad buffer necesaria para neutralizar la acidez y no afectar la DA de los BOM (Abdulkarim y Abdullahi, 2010). En relación con la humedad, el alto contenido (valor superior a $60 \%$ ) puede favorecer la etapa de hidrólisis que ocurre como etapa inicial del proceso de digestión anaerobia (González et al., 2008).

El contenido de materia orgánica de los BOM se eleva, como se evidencia con los valores de $\mathrm{UV}_{254}, \mathrm{COT}, \mathrm{DQO}$, DBO5 y SV, los cuales están asociados a la composición física y fina de los BOM (Li et al., 2010). Adicionalmente, la relación entre la $\mathrm{DQO}_{\text {filtrada }} \mathrm{y}$ la $\mathrm{DQO}_{\text {total }}(0.26)$, indica la predominancia de material particulado que también puede influir sobre la etapa de hidrólisis de la materia orgánica, incrementando el tiempo de retención de sólidos (TRS), como lo evidenciaron Mata et al. (2000) y Castells (2012) en estudios de DA de BOM.

La forma predominante del nitrógeno fue la amoniacal, en la forma de ion amonio ((NH4+) bicarbonato de amonio) y de nitrógeno amoniacal no ionizado
(NH3). El pH influyó sobre la forma predominante del nitrógeno, porque cumple un factor importante en los procesos biológicos, debido a la probable ocurrencia de fenómenos de inhibición de la actividad microbiana (Aldin, 2010), el $\mathrm{NH}_{3}$ es la forma más tóxica y en los BOM la concentración se encontró en niveles bajos $\left(0.038 \mathrm{mg}^{*} \mathrm{~L}^{-1}\right)$ mientras que el $\mathrm{NH}_{4}^{+}$, que aporta alcalinidad bicarbonática, fue de $324.41 \mathrm{mg}^{*} \mathrm{~L}^{-1}$; sin embargo, para la DA de este tipo de residuos, autores como Parawira et al. (2004), recomiendan una concentración del orden de $1100 \mathrm{mg}^{*} \mathrm{~L}^{-1}$ para garantizar buena capacidad buffer.

Los BOM presentaron una relación C/N entre 20-30, lo cual tiene efectos positivos sobre la DA (Juanga, 2005); este valor puede estar relacionado con el alto contenido de proteína. La relación SV/ST y la FB también ratifican el alto contenido de materia orgánica y el bajo contenido de material de origen vegetal de difícil degradación, especialmente la lignina (Takáčová et al., 2012), resultados que son similares a los de Chen et al. (2010).

Generalmente, los BOM presentan contenidos de celulosa y lignina entre $40-60 \%$ y $10-15 \%$, respectivamente (Juanga, 2005); sin embargo, los valores obtenidos fueron inferiores debido a las buenas prácticas de separación en la fuente, pues estos se relacionan con la presencia de papel, pañales desechables y residuos de poda (Delfín y De Bazúa, 2003). Del mismo modo, el contenido de almidón es bajo, posiblemente por el tiempo de almacenamiento en las viviendas (entre 3 y 4 días) que favorece los procesos de fermentación y la 
Tabla 4. Caracterización físico-química de los BOM e inóculo

\begin{tabular}{|c|c|c|}
\hline Parámetro & $\mathrm{BOM}^{* *}$ & Inóculo \\
\hline $\mathrm{pH}$ & 5.54 & 7.23 \\
\hline Alcalinidad total $-\mathrm{AT}\left(\mathrm{CaCO}_{3} \mathrm{mg}^{*} \mathrm{~L}^{-1}\right)$ & 4447.06 & 6270.40 \\
\hline Alcalinidad bicarbonática-ATB $\left(\mathrm{CaCO}_{3} \mathrm{mg}^{*} \mathrm{~L}^{-1}\right)$ & - & 3390.34 \\
\hline Ácidos Grasos Volátiles-AGV $\left(\mathrm{mg}^{*} \mathrm{~L}^{-1}\right)$ & 10595.52 & 1656.58 \\
\hline Humedad (\%) & 76.74 & 94.14 \\
\hline $\operatorname{COT}^{*}(\%)$ & 37.98 & 10.20 \\
\hline Carbono oxidable $(\%)$ & 5.70 & 1.56 \\
\hline $\mathrm{UV}_{254}\left(\mathrm{~cm}^{-1}\right)$ & $>3.30$ & $>3.30$ \\
\hline $\mathrm{DQO}_{\text {total }}\left(\mathrm{mg}^{*} \mathrm{~L}^{-1}\right)$ & 137839.06 & 54852.6 \\
\hline DQO filtrada $\left(\mathrm{mg}^{*} \mathrm{~L}^{-1}\right)$ & 35604.49 & 4048.52 \\
\hline $\mathrm{DBO}_{5}\left(\mathrm{mg}^{*} \mathrm{~L}^{-1}\right)$ & 45333.33 & 1273.89 \\
\hline Nitrógeno total ${ }^{*}(\%)$ & 1.70 & 0.52 \\
\hline Nitrógeno amoniacal $\left(\mathrm{mg}^{*} \mathrm{~L}^{-1}\right)$ & 324.45 & 393.15 \\
\hline Celulosa (\%) & 1.47 & 0.01 \\
\hline Almidón (\%) & $<0.10$ & $<0.10$ \\
\hline Lignina (\%) & 1.20 & 0.01 \\
\hline Extracto etéreo (\%) & 0.96 & 0.50 \\
\hline Proteínas (\%) & 2.70 & 0.71 \\
\hline Carbohidratos (\%) & 9,60 & 0.03 \\
\hline Fibra cruda (\%) & 2.67 & 0.02 \\
\hline Hidrógeno* (\%) & 4.85 & - \\
\hline Oxígeno* (\%) & 32.57 & - \\
\hline Sólidos totales $\left(\mathrm{mg}^{*} \mathrm{~L}^{-1}\right)$ & 113036.67 & 74090 \\
\hline Sólidos volátiles $\left(\mathrm{mg}^{*} \mathrm{~L}^{-1}\right)$ & 93016.67 & 28258.85 \\
\hline
\end{tabular}

Nota: *Base seca**Valores promedio

formación de azúcares. Los otros compuestos como extracto etéreo (lípidos), fibra cruda, proteínas y carbohidratos, se encontraron en niveles similares a los reportados por Chen et al. (2010) para este tipo de residuos.

En relación con el inóculo, presentó valores típicos de lodos anaerobios procedentes del tratamiento de aguas residuales domésticas (ARD), con valores de $\mathrm{pH}, \mathrm{AT}$ y ATB indicativos de una buena capacidad buffer (Pesta, 2007) que favorece su digestión anaerobia; sin embargo, el valor de la relación SV/ST obtenida fue de (0.38), típico de lodos de reactores anaerobios que tratan ARD, el valor es bajo desde el punto de vista del grado de actividad de la biomasa presente en el lodo (Torres et al., 2004). Por otra parte, presentó bajo contenido de materia orgánica, lo cual evidencia las condiciones favorables que aportan los BOM al proceso de DA.

\section{Evaluación de la relación S/I sobre la DA de BOM}

La figura 3 ilustra el PBM durante los 40 días (960 horas) que se llevó a cabo el proceso de DA, la producción de $\mathrm{CH}_{4}$ para las relaciones $\mathrm{S} / \mathrm{I}$ evaluadas varió entre 176.19 y $17.56 \mathrm{~mL} \mathrm{CH}_{4}{ }^{*} \mathrm{~g} \mathrm{SV}^{-1}$ y para el control fue de $27.06 \mathrm{~mL} \mathrm{CH}_{4}^{*} \mathrm{~g} \mathrm{SV}^{-1}$. La producción de $\mathrm{CH}_{4}$ a los 30 días (720 horas), fue superior a 95\% del total para la mayoría de las relaciones $\mathrm{S} / \mathrm{I}$ evaluadas, no obstante, para la 2 y 4 fueron de 78 y $46 \%$, respectivamente. 


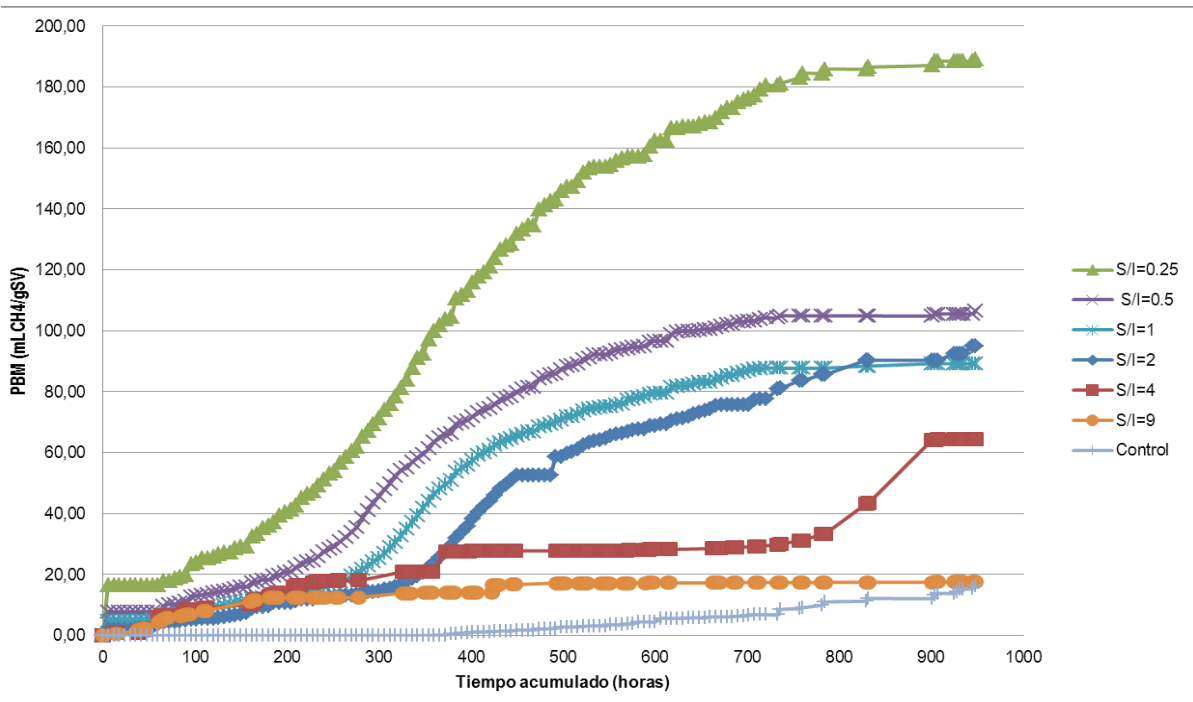

Figura 3. Comportamiento del PBM para las relaciones $S / I$ evaluadas
Se observó que la relación S/I tiene un efecto sobre la fase de latencia, pues en las relaciones menores $(0.25$, $0.5,1$ y 2) esta presentó una duración de 3 a 11 días (72 y 264 horas), mientras que para la relación de 4 fueron 28 días (672 horas) y para la relación de 9 no se observó la transición a la fase intermedia. Los resultados obtenidos coinciden con lo reportado por Chen y Hashimoto (1996), quienes afirman que esta fase es más corta para las relaciones bajas que corresponden a las de menor concentración de sustrato.

En el caso de la relación de 9, no se presentó un incremento significativo en la producción de $\mathrm{CH}_{4}$ y finalizó a los 8 días (192 horas) aproximadamente. En este sentido, Lü et al. (2012) afirman que con el aumento de la relación S/I, hay una menor cantidad de microorganismos disponibles para transformar los productos de la fermentación, lo que puede conducir a sobrecargas debido a la acumulación de AGV's.

La tabla 5 presenta los resultados del PBM total, disuelto y teórico, el porcentaje de metano y el \%B. El primer aspecto a destacar es la efectividad del $\mathrm{NaOH}$ como agente absorbente del $\mathrm{CO}_{2}$, ya que en la composición del biogás predominó el metano.

Estos resultados concuerdan con otros estudios de residuos sólidos, como los de Zhou et al. (2011) y Boulanger et al. (2012). El comportamiento del porcentaje del metano y de biodegradabilidad, ratificaron este efecto, por lo que se encontraron relaciones S/I mayores o iguales a 2, afectando negativamente el proceso anaerobio para las condiciones de este estudio, lo que coincide con autores como Raposo et al. (2006). Este fenómeno se asocia con la inhibición de los consorcios microbianos anaerobios por acción de la acumulación de AGV's, pues se observó que a relaciones S/I mayores de 0.25 comienza a decaer la biodegradabilidad a niveles del orden de 7\% con una relación S/I de 9. Estos resultados se asemejan a los reportados por Zhou et al. (2011), quienes encontraron la mayor producción de metano y porcentaje de biodegradabilidad con relaciones $\mathrm{S} / \mathrm{I}<0.6$.

Otra probable causa del efecto de la relación S/I sobre el PBM es la hidrólisis; según Bouallagui et al. (2005) existe una relación directa entre la materia orgánica soluble (MOS) y la hidrólisis, ya que a mayor contenido de MOS, los tiempos para la formación de sustratos fundamentales en la DA se reducen y la producción de metano se incrementa. En este estudio, el aumento de la relación S/I implicó un aumento de la materia orgánica particulada presente en el sustrato.

Las tablas 6 y 7 muestran los valores $\mathrm{pH}, \mathrm{AT}$, ATB, AGV, I $\alpha$, IB e índice AI/AP para cada una de las relaciones evaluadas al inicio y final del ensayo.

$\mathrm{El}$ incremento de los valores de $\mathrm{pH}$ al final del proceso para las relaciones $\mathrm{S} / \mathrm{I}$ entre 0.25 y 2 , puede asociarse a la ocurrencia de condiciones adecuadas de neutralización de la acidez (capacidad buffer) provocada por la transformación de la materia orgánica en $\mathrm{AGV}^{\prime}$ 's, además de que el inóculo presenta también buena capacidad buffer (López y Beltrán (2005). En el caso de las relaciones $\mathrm{S} / \mathrm{I}$ de 4 y 9, el fenómeno fue el opuesto, debido a la probable acumulación de $\mathrm{AGV}^{\prime}$ s; de acuerdo con Alonso et al. (2006), concentraciones de AGV's cercanas a los $1500 \mathrm{mg}^{*} \mathrm{~L}^{-1}$ pueden desequilibrar el proceso metabólico anaerobio. Este comportamiento se ratifica con los índices $\mathrm{I} \alpha$, IB e índice AI/AP, los cuales fueron estables e indicativos de predominancia de alcalinidad bicarbonática para las relaciones S/I menores que 2; el uso de relaciones mayores, implicaría la necesidad de 
Tabla 5. PBM total, disuelto y teórico, $\% \mathrm{CH}_{4}$ y \%B

\begin{tabular}{cccccc}
\hline $\begin{array}{c}\mathrm{S} / \mathrm{I} \\
\left(\mathrm{gSV}_{\text {sustrato }} \mathrm{gSVV}_{\text {iń́culo }}{ }^{-1}\right)\end{array}$ & $\mathrm{PBM}_{\text {total }}\left(\mathrm{mLCH}_{4}{ }^{*} \mathrm{gSV}^{-1}\right)$ & $\mathrm{PBM}^{*}\left(\mathrm{mLCH}_{4}{ }^{*} \mathrm{gSV}^{-1}\right)$ & $\% \mathrm{CH}_{4}$ & $\begin{array}{c}\mathrm{PBM}_{\text {teórico }} \\
\left(\mathrm{mLCH}_{4}^{*} \mathrm{gSV}^{-1}\right)\end{array}$ & $\%^{\circ B}$ \\
\hline 0.25 & $176.19 \pm 18.37$ & $19.3 \pm 2.18$ & 99.99 & 240 & 73.12 \\
0.5 & $106.25 \pm 0.47$ & $11.7 \pm 0.05$ & 99.99 & 240 & 44.09 \\
1 & $101.75 \pm 17.66$ & $11.2 \pm 1.94$ & 99.89 & 240 & 42.33 \\
2 & $90.88 \pm 12.54$ & $9.7 \pm 1.01$ & 99.05 & 240 & 37.72 \\
4 & $75.04 \pm 15.10$ & $8.2 \pm 1.64$ & 90.97 & 240 & 31.14 \\
9 & $17.56 \pm 0.52$ & $1.8 \pm 0.19$ & 79.81 & 240 & 7.29 \\
Control & $27.06 \pm 15.43$ & $3.0 \pm 1.70$ & 99.20 & N.D & N.D \\
\hline
\end{tabular}

* N.D: No determinado; *PBM considerando únicamente el metano disuelto

Tabla 6. Valores de pH, AT, ATB, AGV, I $\alpha$, IB e Índice Al/AP al inicio del ensayo

\begin{tabular}{cccccccc}
\hline $\mathrm{S} / \mathrm{I}^{*}$ & $\mathrm{pH}(\mathrm{UND})$ & $\mathrm{AT}\left(\mathrm{mg} \mathrm{CaCO}_{3}{ }^{*} \mathrm{~L}^{-1}\right)$ & $\mathrm{ATB}\left(\mathrm{mg} \mathrm{CaCO}_{3}{ }^{*} \mathrm{~L}^{-1}\right)$ & $\mathrm{AGV}\left(\mathrm{mg}^{*} \mathrm{~L}^{-1}\right)$ & $\mathrm{I} \alpha$ & $\mathrm{IB}$ & $\mathrm{AI} / \mathrm{AP}$ \\
\hline 0.25 & 7.04 & 332.86 & 240.88 & 700.45 & 0.72 & 0.28 & 0.38 \\
0.5 & 7.03 & 378.49 & 264.83 & 694.20 & 0.70 & 0.30 & 0.42 \\
1 & 7.04 & 380.75 & 251.88 & 896.31 & 0.66 & 0.34 & 0.52 \\
2 & 7.11 & 368.32 & 186.83 & 1119.48 & 0.51 & 0.49 & 1.06 \\
4 & 7.00 & 707.55 & 374.38 & 1826.18 & 0.53 & 0.47 & 0.90 \\
9 & 7.01 & 1234.64 & 549.70 & 2626.70 & 0.45 & 0.55 & 1.24 \\
Control & 7.13 & 244.48 & 161.86 & 612.90 & 0.66 & 0.34 & 0.52 \\
\hline
\end{tabular}

*(gSV sustrato $\left.^{*} \mathrm{gSV}_{\text {inóculo }}{ }^{-1}\right)$

Tabla 7. Valores de pH, AT, ATB, AGV, I $\alpha$, IB e Índice Al/AP al final del ensayo

\begin{tabular}{cccccccc}
\hline $\mathrm{S} / \mathrm{I}^{*}$ & $\mathrm{pH}(\mathrm{UND})$ & $\mathrm{AT}\left(\mathrm{mg} \mathrm{CaCO}_{3}{ }^{*} \mathrm{~L}^{-1}\right)$ & $\mathrm{ATB}\left(\mathrm{mg} \mathrm{CaCO}_{3}{ }^{*} \mathrm{~L}^{-1}\right)$ & $\mathrm{AGV}\left(\mathrm{mg}^{*} \mathrm{~L}^{-1}\right)$ & $\mathrm{I} \alpha$ & $\mathrm{IB}$ & $\mathrm{AI} / \mathrm{AP}$ \\
\hline 0.25 & 10.32 & 2188.72 & 1720.70 & 1538.50 & 0.79 & 0.21 & 0.31 \\
0.5 & 9.29 & 1119.00 & 736.56 & 1444.69 & 0.65 & 0.34 & 0.55 \\
1 & 8.65 & 1498.20 & 1030.82 & 1357.13 & 0.68 & 0.34 & 0.48 \\
2 & 7.48 & 1314.05 & 738.29 & 1626.05 & 0.56 & 0.43 & 0.78 \\
4 & 6.29 & 1450.11 & 413.02 & 2945.66 & 0.28 & 0.72 & 2.52 \\
9 & 4.99 & 871.77 & 0.00 & 7304.74 & 0.00 & 1.00 & - \\
Control & 11.4 & 1523.01 & 1231.25 & 612.90 & 0.81 & 0.19 & 0.24 \\
\hline
\end{tabular}

$*\left(\mathrm{gSV}_{\text {sustrato }} \mathrm{gSV}_{\text {inóculo }^{-1}}\right)$

mayores cantidades de acondicionador químico que garantice capacidad buffer, lo que incrementaría los costos y la complejidad operacional.

\section{Conclusiones y recomendaciones}

- Los biorresiduos BOM presentaron una composición predominante de materia orgánica y suspendida, con niveles de nutrientes adecuados para garantizar un buen desempeño del proceso anaerobio; sin embargo, los bajos niveles de $\mathrm{pH}$ y alcalinidad bicarbo- nática, asociado a las altas concentraciones de AGV, indican la necesidad de acondicionar el sustrato con un alcalinizante.

- La relación S/I influyó sobre la digestión anaerobia de los BOM, se encontró que relaciones menores a 2 $\mathrm{gSV}_{\text {sustrato }}{ }^{*} \mathrm{gSV}_{\text {inóculo }}{ }^{-1}$ permiten un desempeño adecuado del proceso; con valores mayores, el proceso se desestabiliza debido a la ocurrencia de procesos de acidificación por la acumulación de los AGV's que no logran ser estabilizados por la baja capacidad buffer del sistema. En consecuencia, se deben garanti- 
zar condiciones de $\mathrm{S} / \mathrm{I}$ y $\mathrm{pH}$ que permitan menores tiempos de retención y estabilidad durante el proceso de transformación del sustrato.

- El posible fenómeno de inhibición ocurrido en las relaciones mayores a $2 \mathrm{gSV}_{\text {sustrato }}{ }^{*} \mathrm{gSV}_{\text {inóculo }}{ }^{-1}$, puede estar asociado a la alteración de la etapa hidrolítica, ya que de esta fase de la DA depende el éxito de degradación de la materia orgánica y por ende, de la producción de metano.

- Debido a las condiciones favorables que propiciaron los BOM al proceso de DA, se recomienda evaluar la incidencia de la adición de macro y micro nutrientes al proceso, con el fin de observar su efecto sobre la producción de metano.

\section{Agradecimientos}

Los autores agradecen a la Universidad del Valle por la financiación del proyecto de investigación "Evaluación del potencial de producción de energía a partir de la digestión anaerobia de biorresiduos de origen municipal-CI 2704", a la Cooperativa de Servicios Públicos de Versalles, Camino Verde APC y a EMCALI EICE ESP, por el apoyo para el desarrollo de este estudio.

\section{Referencias}

Abdulkarim B.I., Abdullahi M.E. Effect of buffer $\left(\mathrm{NaHCO}_{3}\right)$ and waste type in high solid thermophilic anaerobic digestion. International Journal of ChemTech Research, volumen 2 (número 2), 2010: 980-984.

Aldin S. The effect of particle size on hydrolysis and modeling of anaerobic digestion, tesis (doctor en ingeniería), Canada, Universidad de Western Ontario, 2010, 230 p.

Alonso V.C., Gómez L.C., García H.F., Rodríguez M.J.M. Producción de biogás a partir de residuos vegetales. Ingeniería Química, (número de febrero) 2006: 114-126.

Angelidaki I., Alves M., Bolzonella D., Borzacconi L., Campos J.L., Guwy A.J., Kalyuzhnyi S., Jenicek P., Van-Lier J.B. Defining the Biomethane Potential (BMP) of solid organic wastes and energy crops: A proposed protocol for batch assays. Water Science \& Technology, volumen 59 (número 5), 2009: 927-34.

Angelidaki I. y Sanders W. Assessment of the anaerobic biodegradability of macropollutants. Reviews in Enviromental Sciencia and BioTechnology, (número 3), 2004: 117-29.

APHA. Standard methods for examination of water and wastewater, Washington DC, American Water Works Association and Water Environment Federation, 2005.

Aquino S.F. Influência Das Condições De Incubação No Teste De Atividade Metanogênica Específica (AME) De Lodos Anaeróbios, tesis (maestría en ingeniería), Brasil, Universidad Federal de Minas Gerais, 2007, 189 p.

Aquino S.F., Chernicharo L.C.A., Foresti E., Florencio D.S.M. Metodologias para Determinação da Atividade Metanogênica Específica (AME) em Lodos Anaeróbios. Eng. Sanit. Ambient., volumen 12, 2007: 192-201.

Bouallagui H., Touhami Y., Ben C.R., Hamdi M. Bioreactor performance in anaerobic digestion of fruit and vegetable wastes. Process Biochemistry, volumen 40 (número 3), 2005: 989-95.

Boulanger A., Pinet E., Bouix M., Bouchez T., Mansour A.A. Effect of inoculum to substrate ratio (i/s) on municipal solid waste anaerobic degradation kinetics and potential. Waste Management, volumen 32, 2012: 2258-65.

Castells X.E. Métodos de valorización y tratamiento de residuos municipales, Reciclaje de Residuos Industriales, ediciones Díaz de Santos, 2012, $866 \mathrm{p}$.

Charles W., Walker L., Cord-Ruwisch R.. Effect of pre-aeration and inoculum on the start-up of batch thermophilic anaerobic digestion of municipal solid waste. Bioresour Technol., volumen 100, 2009: 2329-2335.

Chen T.H., Hashimoto A.G. Effects of $\mathrm{pH}$ and substrate: inoculum ratio on batch methane fermentation. Bioresource Technology, volumen 56 (número 2), 1996: 179-186.

Chen X., Romano R.T., Zhang R. Anaerobic digestion of food wastes for biogas production. Int. J. Agric. E Biol. Eng., volumen 3 (número 4), 2010: 61-71.

Delfín A.I., De Bazúa D.C. Biodegradación de residuos urbanos lignocelulósicos por Pleurotus. Rev. Int. Contam. Ambient., volumen 19 (número 1), 2003: 37-45.

Espinosa Ll.M. del C., López T.M., Pellón A.A., Mayarí N.R., Fernández C.A. La fracción orgánica de los residuos sólidos urbanos como fuente potencial de producción de biogás. Revista CENIC Ciencias Biológicas, volumen 38 (número 1), 2007: 33-37.

Field J. Arranque y operación de sistemas de flujo ascendente con manto de Lodo-UASB. Universidad del Valle, CVC, Universidad Agrícola de Wageningen, 1987.

Filigrana P.A., Gómez O.L., Méndez F. Impacto de un sitio de disposición final de residuos sólidos en la salud respiratoria de los adultos mayores. Biomédica, volumen 31, 2011: 322-34.

Giménez J.B., Martí N., Ferrer J., Seco A. Methane recovery efficiency in a submerged anaerobic membrane bioreactor (SANMBR) treating sulphate-rich urban wastewater: Evaluation of methane losses with the effluent. Bioresoruce Technology, volumen 118, 2012: 67-72.

González G.I., Rustrián E., Houbron E., Zamora A. Impacto de la tasa de humedad en la biodegradación de los residuos sólidos urbanos de la ciudad de Veracruz, México. Revista Latinoamericana de Recursos Naturales, volumen 4 (número 3), 2008: 336-41.

Grajales A.C. Análisis de la variabilidad de la calidad de los sustratos del compostaje de biorresiduos en la cabecera municipal de versallesvalle del cauca, acorde con el día de montaje, tesis (pregrado en ingeniería), Colombia, Universidad del Valle, 2012, 59 p. 
ICONTEC. Norma Técnica Colombiana 5167. Productos para la industria agrícola, productos orgánicos usados como abonos o fertilizantes y enmiendas de suelo, 2004, 32 p.

ICONTEC. Norma Técnica Colombiana 1369. Determinación de Boro, Calcio, Cobalto, Cobre, Hierro, Magnesio, Manganeso, Molibdeno, Níquel, Silicio y Zinc por absorción atómica, 2009, $3 p$.

Juanga J.P. Optimizing dry anaerobic digestion of organic fraction of municipal solid waste, tesis (maestría in ingeniería), Tailandia, Asian Institute of Technology, 2005, $160 \mathrm{p}$.

Lesteur M., Bellon-Maurel V., Gonzalez C., Latrille E., Roger J.M., Junqua G., Steyer J.P. Alternative methods for determining anaerobic biodegradability: A Review. Process Biochem, volumen 45, 2010: 431-40.

Li R., Chen S., Li X. Biogas production from anaerobic co-digestion of food waste with dairy manure in a two-phase digestion system. Applied Biochemistry and Biotechnology, volumen 160 (número 2), 2010: 643-54.

López M.G., Beltrán R. Digestión anaerobia de residuos sólidos orgánicos urbanos. Estimación del tiempo de retención dependiendo de la concentración de sólidos volátiles totales y de la presencia o no de un inóculo [en línea] [fecha de consulta: 15 de febrero de 2013] Disponible en: http://dspace.uniandes.edu.co/ xmlui/bitstream/handle/1992/226/mi_1197.pdf?sequence=1.

Lü F., Hao L., Zhu M., Shao L., He P. Initiating methanogenesis of vegetable waste at low inoculum-to-substrate ratio: importance of spatial separation. Bioresource Technology, volumen 105, 2012:169-173.

Marmolejo L.F. Marco conceptual para el aprovechamiento en plantas de manejo de residuos sólidos de poblaciones menores a 20.000 habitantes del Norte del Valle del Cauca-Colombia, tesis (doctorado en ingeniería), Colombia Universidad del Valle, 2011, 190 p.

Mata A.J., Macé S., Llabares P. Anaerobic digestion of organic solid wastes. An overview of research achievements and perspectives. Bioresoruce Technology, volumen 74, 2000: 3-16.

Mukherjee S., Kumar S., Devotta. S. Influence of Nitrogen of anaerobic digestion of municipal solid easte in a laboratory scale reactor. Journal of the IPHE, volumen 09 (número 4), 2008: 19-24.

Ortiz J.V. Puesta a punto de una metodología para la determinación de la actividad metanogénica específica (ame) de un fango anaerobio mediante el sistema Oxitop ${ }^{\circledR}$. Influencia de las principales variables experimentales, tesis (maestría en ingeniería), España, Universidad Politecnica de Valencia, 2011, 110 p.

Oviedo E.R., Daza, T.M.C., Marmolejo L.F., Osorio O.A., Torres L.P. Influencia de la incorporación de pasto estrella como material de soporte (Cynodon Plectostachyus) en el compostaje de biorresiduos de origen municipal. Ingeniería y Desarrollo, volumen 31 (número 2), 2013: 252-71.

Oviedo O.R., Marmolejo L.F., Torres L.P. Perspectivas de aplicación del compostaje de biorresiduos provenientes de residuos sólidos municipales. Un enfoque desde lo global a lo local.
Revista Ingenierías Universidad de Medellín, volumen 11 (número 20), 2012: 67-76.

Owen W.F, Stuckey C.D., Healy J.B., Young L.Y., McCarty P.L. Bioassay for monitoring biochemical methane potential and anaerobic toxicity. Water Research, volumen 13, 1978: 485-492.

Pabón P.C.P., Castañares G., Van Lier J.B. An Oxitop ${ }^{\circledR}$ protocol for screening plant material for its Biochemical Methane Potential (BMP). Water Science and Technology, volumen 66 (número 7), 2012: 1416-23.

Parawira W., Murto M., Zvauya R., Mattiasson B. Anaerobic batch digestion of solid potato waste alone and in combination with sugar beet leaves. Renewable Energy, volumen 29 (número 18), 2004: 11-1823.

Parfitt J., Barthel M., Macnaughton S. Food waste within food supply chains: quantification and potential for change to 2050 . Philosophical transactions of the royal society B. Biological Sciences, volumen 365 (número 1554), 2010:3065-81.

Pesta G. Anaerobic digestion of organic residues and wastes New York, Springer US, 2007.

Raposo F., Banks C.J., Siegert I., Heaven S., Borja R. Influence of inoculum to substrate ratio on the biochemical methane potential of maize in batch tests. Process Biochemistry, volumen 41, 2006: 1444-50.

Raposo F., Fernández C.V., De la Rubia M.A., Borja R., Béline F., Cavinato C., Demirer G. Biochemical Methane Potential (BMP) of solid organic substrates: Evaluation of anaerobic biodegradability using data from an international interlaboratory study. Journal of Chemical Technology and Biotechnology, volumen 86 (número 8), 2011: 1088-98.

Sakurai K. Método sencillo del análisis de residuos sólidos, CEPIS, Lima-Peru: CEPIS/OPS, 2000.

Sandoval C., Carreño M., Castillo E.F., Vergara M. Caracterización microbiológica de los lodos que intervienen en la digestión anaerobia de la fracción orgánica de los residuos sólidos urbanos. Revista ION, volumen 20, (número 1), 2007: 58-62.

Sharma K.S., Mishra I.M., Sharma M.P., Saini, J.S. Effect of particle size on biogas generation from biomass residues. Biomass, volume 17, 1988: 251-263.

Siefers A.M.A Novel and cost-effective hydrogen sulfide removal technology using tire derived rubber particles, tesis (maestro en ciencia), United State, Iowa State University, 2010, 85 p.

Sobotka M., Votruba J., Havlík I., Minkevich, I.G. The mass-energy balance of anaerobic methane production. Folia Microbiologica, volumen 28 (número 3), 1983: 195-204.

Takáčová A., Macklul'ak T., Smolinská M., Hutňan M., Olejníková P.. Influence of selected biowaste materials pre-treatment on their anaerobic digestion. Chemical Papers, volumen 66 (número 2), 2012: 129-37.

Torres L.P., Pérez A. Actividad metanogénica específica: una herramienta de control y optimización de sistemas de tratamiento anaerobio de aguas residuales. Revista EIDENAR, (número 9), 2010: 5-14. 
Torres L.P., Pérez A. Índices de alcalinidad para el control del tratamiento anaerobio de aguas residuales fácilmente acidificables. Ingeniería y Competitividad, volumen 10 (número 2), 2008: 41-52.

Torres P., Cardoso A., Rojas O. Mejoramiento de la calidad de lodos anaerobios. Influencia de la adición de Cloruro Férrico. Ingeniería y Competitividad, volumen 5 (número 2), 2004: 23-31.

TWB. World Production of Municipal Solid Waste (MSW), 2012*2025. The Wordl Bank, 2012.

VDI. Fermentation of organic materials characterisation of the substrate, sampling, collection of material data, fermentation tests, Alemania, Verein Deustcher Ingenieure, 2006.

Verma S. Anaerobic digestion of biodegradable organics in municipal solid wastes, tesis (maestría en ciencia), EU, Columbia University, 2002, $50 \mathrm{p}$.

Zhou Y., Zhang Z., Nakamoto T., Li Y., Yang Y., Utsumi M., Sugiura $\mathrm{N}$. Influence of substrate-to-inoculum ratio on the batch anaerobic digestion of bean curd refuse-okara under mesophilic conditions. Biomass and Bioenergy, volumen 35, 2011: 3251-56.

\section{Este artículo se cita:}

\section{Citación estilo Chicago}

Parra-Orobio, Brayan Alexis, Patricia Torres-Lozada, Luis Fernando Marmolejo-Rebellón, Lina Marcela Cárdenas-Cleves, Carlos Vásquez-Franco, Wilmar Alexander Torres-López, José Abdón Ordoñez-Andrade. Efecto de la relación sustrato-inóculo sobre el potencial bioquímico de metano de biorresiduos de origen municipal. Ingeniería Investigación y Tecnología, XVI, 04 (2015): 515-526.

\section{Citación estilo ISO 690}

Parra-Orobio B.A, Torres-Lozada P, Marmolejo-Rebellón L.F, Cardenas-Cleves L.M, Vásquez-Franco C., Torres-López W.A., Ordoñez-Andrade J.A Efecto de la relación sustrato-inóculo sobre el potencial bioquimico de metano de biorresiduos de origen municipal. Ingeniería Investigación y Tecnología, volumen XVI (número 4), octubre-diciembre 2015: 515-526.

\section{Semblanzas de los autores}

Brayan Alexis Parra-Orobio: Ingeniero sanitario, magíster en ingeniería (ingeniería sanitaria y ambiental) por la Universidad del Valle, Cali, Colombia. Se desempeñó durante 6 meses como asistente de investigación en el grupo de investigación: "Estudio y control de la contaminación ambiental-ECCA" de la Universidad del Valle. Actualmente es estudiante de doctorado en ingeniería en la misma institución.

Patricia Torres-Lozada: Ingeniera sanitaria por la Universidad del Valle, es magister y doctora en ingeniería civil (hidrualica y sanemiento) por la Universidad São Paulo, Brasil. Profesora titular de la Universidad del Valle. Directora del grupo de invesitgacion: "Estudio y control de la contaminación ambiental-ECCA" por la Universidad del Valle. Trabajó durante más de 10 años en temas relacionados con el tratamiento de aguas residuales doméstias e industriales, manejo de residuos sólidos y sistemas de potabilización. Es Investigadora Senior, máxima categoría otorgada por del Departamento Administrativo de Ciencia, Tecnología e Innovación-Colciencias.

Luis Fernando Marmolejo-Rebellón: Ingeniero sanitario, magister en admistración en salud y doctor en ingeniería por la Universidad del Valle, Cali, Colombia. Profesor asistente en la Universidad del Valle. Se desempeñó como líder de la línea de investigación de manejo y aprovechamiento de los residuos sólidos municipales desde 1999 en el Grupo de Investigación: "Estudio y control de la contaminación ambiental-ECCA" en la Universidad del Valle. Es investigador asociado de acuerdo con el Departamento Administrativo de Ciencia, Tecnología e Innovación-Colciencias.

Lina Marcela Cárdenas-Cleves: Ingeniera Sanitaria de la Universidad del Valle, Cali, Colombia. Ha trabajado como consultora en el área de la ingeniería, particularmente enfocada a solucionar problemas ambientales en el Valle del Cauca, Colombia. Actualmente es estudiante de Maestría en Ingeniería énfasis en Ingenieria Sanitaria y Ambiental además es Asistente de Investigación en el Grupo de Investigación: "Estudio y Control de la Contaminación Ambiental-ECCA" de la Universidad del Valle.

Carlos Vásquez-Franco: Es ingeniero sanitario por la Universidad del Valle, Cali, Colombia. Se desempeñó como consultor en temas relacionados con saneamiento ambiental en el Valle del Cauca, Colombia. Actualmente es estudiante de especialización en ingeniería sanitaria y ambiental de la Universidad del Valle.

Wilmar Alexander Torres-López: Estadístico, estudiante de maestría en estadística en la Universidad del Valle, Cali, Colombia. Es profesor auxiliar en la misma institución y miembro del grupo de investigación: "Estadística aplicada-INFERIR" de la Universidad del Valle, donde ha trabajado en proyectos de investigación relacionados a la modelación y series de tiempo en temas ambientales.

José Abdón Ordoñez-Andrade: Ingeniero sanitario e industrial de la Universidad del Valle, Cali, Colombia. Magister en ingeniería de procesos por la Universidad de Sttugart y candidato a doctor en ingeniería por la Universidad de Kassel, Alemania. Durante más de 5 años ha trabajado en proyectos de investigación relacionados con el tratamiento de aguas residuales, potabilización y manejo de residuos sólidos. Actualmente realiza una investigación doctoral enfocada a procesos de filtración avanzados para agua potable en comunidades rurales de países en desarrollo. 